\title{
A High-Density L-Shaped Backscattering Chipless Tag for RFID Bistatic Systems
}

\author{
Khaled Issa $\mathbb{D}^{1}{ }^{1}$ Yazeed A. Alshoudokhi, ${ }^{2}$ Muhammad A. Ashraf $\mathbb{D}^{1}{ }^{1}$ \\ Mohammed R. AlShareef, ${ }^{3}$ Hatim M. Behairy $\mathbb{D}^{3}{ }^{3}$ S. Alshebeili, ${ }^{1,2}$ and H. Fathallah $\mathbb{D}^{1,4}$ \\ ${ }^{1}$ KACST-TIC in Radio Frequency and Photonics for the e-Society (RFTONICS), Electrical Engineering Department, \\ King Saud University, Riyadh, Saudi Arabia \\ ${ }^{2}$ Department of Electrical Engineering, College of Engineering, King Saud University, Riyadh, Saudi Arabia \\ ${ }^{3}$ National Center for Electronics, Communication and Photonics, King Abdulaziz City for Science and Technology, \\ Riyadh, Saudi Arabia \\ ${ }^{4}$ Computer Department, College of Science of Bizerte, Carthage University, Tunis, Tunisia
}

Correspondence should be addressed to Khaled Issa; kissa@ksu.edu.sa

Received 21 March 2018; Accepted 19 June 2018; Published 1 August 2018

Academic Editor: Luca Catarinucci

Copyright (C) 2018 Khaled Issa et al. This is an open access article distributed under the Creative Commons Attribution License, which permits unrestricted use, distribution, and reproduction in any medium, provided the original work is properly cited.

\begin{abstract}
Chipless radiofrequency identification (RFID) technology is very promising for sensing, identification, and tracking for future Internet of Things (IoT) systems and applications. In this paper, we propose and demonstrate a compact 18-bit, dual polarized chipless RFID tag. The proposed tag is based on L-shaped resonators designed so as to maximize the spectral and spatial encoding capacities. The proposed RFID tag operates an over $4 \mathrm{GHz}$ frequency band (i.e., $6.5 \mathrm{GHz}$ to $10.5 \mathrm{GHz}$ ). The tag is simulated, fabricated, and tested in a nonanechoic milieu. The measured data have shown good agreement with the simulation results, with respect to resonators' frequency positions, null depth, and null bandwidth over the operating spectrum. The proposed design achieves spectral and spatial encoding capacities of $4.5 \mathrm{bits} / \mathrm{GHz}$ and $18.8 \mathrm{bits} / \mathrm{cm}^{2}$, respectively. This, in turn, gives an encoding density of $4.7 \mathrm{bits} / \mathrm{GHz} / \mathrm{cm}^{2}$. For code identification, we exploit the frequency content of the backscattered signals and identify similarity/correlation features with reference codes.
\end{abstract}

\section{Introduction}

The huge spread of new smart devices and objects is leading to an exponential growth in the control and management of the worldwide infrastructures. The Internet of Things (IoT) is the focus of a deep global investigation and exploration with the objective of connecting an outsized number of "things"such as smart sensors, tags, and detectors [1-6]. Indeed, automated sensing, identification, and physical tracking of objects are essential and fundamental for the development of modern electronic society.

These innovative devices and instruments (sensors, tags, detectors, etc.) will be capable of delivering information describing their milieu and sharing it using wireless communications [7]. The next-generation radiofrequency identification (RFID) technology, known as chipless RFID, may offer a very attractive solution in this regard [5-8]. In fact, the chipless RFID is based on a wireless data-capturing procedure that exploits the RF energy or signature to detect, extract, and read automatically the characteristics of remotely located "things" [9]. Hence, the RFID technology is one of the most advanced and quickly emerging technologies that have the potential to make abundant economic advancement in many industries [10].

The chipless RFID tag has the potential to replace the barcode because of a number of advantages, comprising the non-line-of-sight reading capability, cost, and data rate [11]. Chipless RFID systems consist of readers and tags. This tag can be well thought-out as a radar target with a particular electromagnetic signature used for coding. The work principles of such tags are based on the retransmission or electromagnetic backscattering. The defies in the design of chipless 
RFID tags are the requirements to be of low cost and of high capacity [12-14]. The main challenge in the design of chipless tags is the manageable area density, also called the spatial capacity of stockpiled data (in bits per $\mathrm{cm}^{2}$ ) [14]. Chipless RFID tags are designed so as to respond to an interrogating wave, which may have vertical, horizontal, circular, or elliptical polarization. Depending on the desired application, a specific polarization can be used. In the literature, the information is encoded using frequency, time, or hybrid frequency-time encoding techniques [15-18].

In this study, we consider frequency domain- (FD-) based tags, which have higher data capacity compared to other encoding techniques $[19,20]$. The fundamental concept of the frequency coding technique is to control the presence or absence of a resonance at particular frequencies of the spectrum. The presence of particular resonance frequencies appears as notches or nulls in the detected/received tag responses. That is, the existence of a null in the signature is attributed to one ( $b i t=1)$ and its absence is attributed to zero (bit $=0$ ). Both the "zero" and "one" digits are considered as elementary bits in the signature. Each resonance is related to a physical scatterer, and the coding capacity is then associated with the number of resonators existing in the tag and in the allowed frequency band [21]. Therefore, the number of codes can be exponentially increased by simply increasing the number of bits.

Chipless FD tags are designed using frequency-selective surfaces (FSS) for encoding and backscattering the interrogating wave. Two different interrogation techniques are often employed at the RFID reader. The first uses a single antenna to interrogate and read the backscattered wave (monstatic system). The second technique, on the other hand, has both an interrogator antenna and a reader antenna (bistatic measurement). This latter technique, which is the focus of our study, has been considered in several publications, including [21-34]. Note that the shape of resonators in FSS-based tags mainly governs the spectral encoding capacity. Chipless tags with patch-based resonators of square loop, U, V, C, and I shapes have been designed and demonstrated in the literature [21-34]. A dual-polarization coding method is a practical alternative in the quest for higher data capacities in FD chipless RFID tags. An enhanced encoding capacity can be accomplished by using two orthogonal polarizations for tag interrogation. This technique is used to advance the data content, while it is still encoded in the frequency domain.

This work presents a novel UWB chipless RFID FD tag, which makes use of L-shaped multiresonators for bistatic systems. L-shaped chipless RFID tags have recently been considered but for monstatic systems [35]. It is relevant at this point to mention that for monstatic systems, an infinite array of identical tags is first designed and then truncated to a typically $3 \times 3$-unit array $[6,19]$, which inevitably increases the size of the end-product tag. On the other hand, designing a tag for bistatic systems is based on the concept of plane wave excitation. This design method ends up with a single unit tag of much reduced size as compared to the truncated array intended for monstatic systems. Another advantage of using bistatic systems is that they generally deliver better performance in terms of sensitivity and dynamic range than monostatic systems do. This is intuitively not surprising because two dedicated antennas are used in such systems to send and receive signals, while only one antenna is used for signal transmission and reception in monostatic systems [6].

The proposed L-shaped resonators have attractive advantages in that they can generate horizontally and vertically polarized signals. Moreover, resonators' lengths can be varied in the horizontal and vertical directions to have independent spectral signatures in both polarizations. The proposed circuit is designed and optimized using the CST simulation program. A set of 1024 different codes is simulated, and their frequency domain responses are recorded under both horizontal and vertical polarizations. These codes are distinct, as will be demonstrated by computing the similarities among FD spectral signatures using signal processing-based correlation methods. The proposed encoding/identification method is validated experimentally by fabricating two RFID tags operating over the proposed spectrum. The experimental characterization of the proposed system is done in an nonanechoic chamber using the vector network analyzer (VNA) acting as an RFID transceiver. The fabricated circuits have shown excellent performance having 18 bits presented by deep nulls ranging from 9 to $14.5 \mathrm{~dB}$ over the considered spectrum and encoding density (D) of $4.7 \mathrm{bits} / \mathrm{GHz} / \mathrm{cm}^{2}$. According to the authors' knowledge, this is the highest encoding density achieved so far for chipless RFID tags characterised using bistatic type of measurements.

\section{Proposed Chipless Tag}

The main goal of this work is to increase the number of bits of a chipless tag while minimizing its size for a particular frequency range. The main methodology relies on coding the data in order to get a dissimilarity between vertically and horizontally polarized responses of a chipless tag. The selectivity of the frequency-domain response is realized via a periodic structure. The proposed layout is optimized to increase the density along with the performance of the chipless RFID tag. The proposed design is depicted in Figure 1, which consists of a stack of $10 \mathrm{~L}$-shaped resonators. The critical design parameters $L_{\mathrm{g}}, W_{\mathrm{g}}, L_{i}^{\mathrm{V}}, L_{i}^{\mathrm{H}}, W, \Delta^{\mathrm{V}}$, and $\Delta^{\mathrm{H}}$ are, respectively, the ground length and width, the length of the $i$ th resonator in the vertical and horizontal directions $(i=1,2, . ., N)$, the slot width, and the vertical and horizontal spacings.

This periodic $10 \mathrm{~L}$-shaped structure is optimized to get deep resonant notches at desired frequencies. We initialize the design process with optimizing two resonators and then reoptimizing after the addition of a new resonator till we develop the whole structure. The two first resonators originate the first resonance frequency or null (lowest and closest to $6.5 \mathrm{GHz}$ ) in the operating frequencies (from 6.5 to $10.5 \mathrm{GHz}$ ). This null represents the lowest significant bit of the code. Essentially, in the proposed design, the length of the $j$ th horizontal/vertical patch decreases as the value of $i$ increases $(i=1,2, . ., N)$, according to the L-shaped structure. This inherently leads to the creation of $N-1$ nulls or code of $(N-1$ bits). The simulation is 


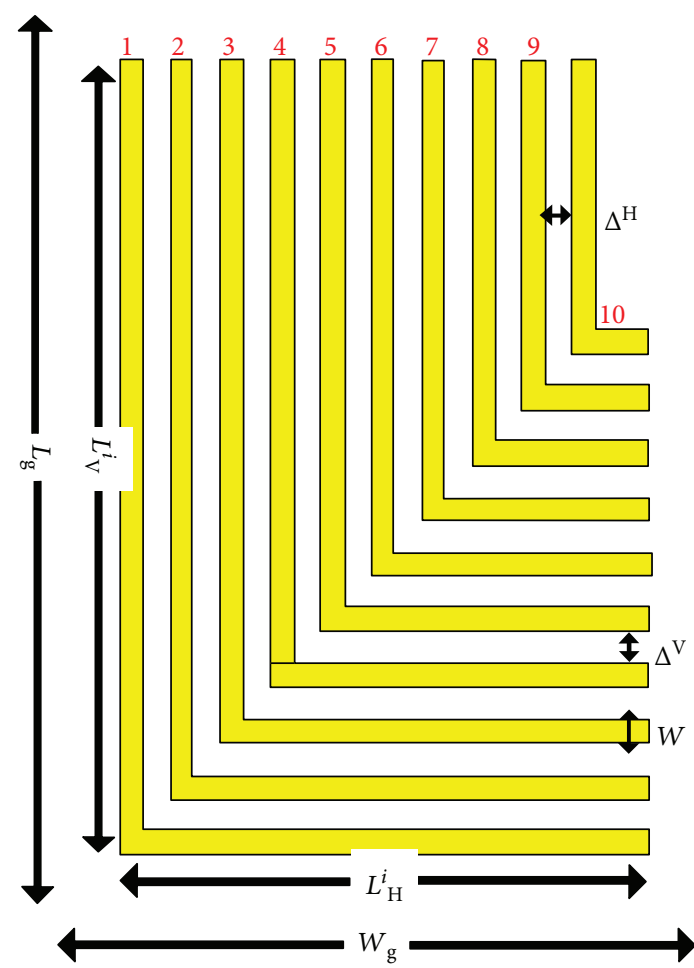

(a)

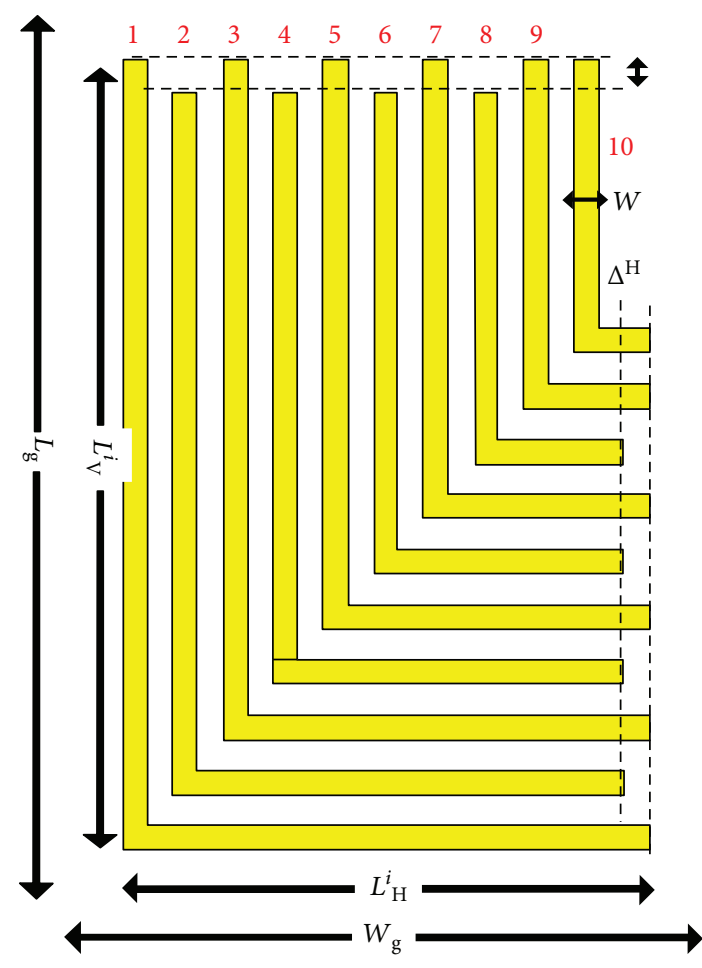

(b)

FIgURE 1: Structure of the proposed L-shaped chipless tag: (a) code 111111111 and (b) code 010101010.

made using full-wave simulation based on the wave plane excitation of CST Microwave Studio (MWS). The proposed chipless tag is fabricated on a single-layer dielectric substrate. The dielectric substrate is low-loss Rogers Duroid RT5880 having a loss tangent of $\tan \delta=0.002$, a dielectric constant of $\epsilon_{r}=2.2$, and a thickness of $h=0.56 \mathrm{~mm}$. We have used copper as a metal with a thickness $t=0.018 \mathrm{~mm}$. Different frequency signatures are generated by performing different optimizations of the parameters of L-shaped resonators. We suggest the following key steps for optimizing the backscattered chipless L-shaped RFID tag:

(1) The design process is initiated as follows. We firstly determine the maximum length of the resonator in the vertical and horizontal directions $L_{1}^{\mathrm{V}}$ and $L_{1}^{\mathrm{H}}$, which would obviously limit the maximum length that can be considered for all other elements. In order to determine this length, we use (1) and (2):

$$
\begin{aligned}
& f_{1}^{\mathrm{H}}=\frac{c}{2 L_{1}^{\mathrm{H}}} \sqrt{\frac{2}{1+\epsilon_{r}}}, \\
& f_{1}^{\mathrm{V}}=\frac{c}{2 L_{1}^{\mathrm{V}}} \sqrt{\frac{2}{1+\epsilon_{r}}},
\end{aligned}
$$

where $f_{1}^{\mathrm{H}}$ and $f_{1}^{\mathrm{V}}$ are the horizontal and vertical resonant frequencies, respectively. The parameters $c$ and $\epsilon_{r}$ represent the speed of light and the relative permittivity of the substrate, respectively. Different frequency signatures are generated using different lengths of L-shaped resonators. We notice that the effective horizontal and vertical lengths are reduced through the introduction of an additional resonator.

(2) We optimize the structure to get a higher number of range deep notches, presenting different resonant frequencies, with a narrow bandwidth within a limited tag size and for a particular frequency band. We note that increasing the encoding density is essentially based on increasing the number of bits and reducing the tag size, simultaneously, for a fixed frequency band. In order to double the encoding capacity, the L-shaped resonator-based structure is optimized to get different spectral signatures in both horizontal and vertical polarizations. Therefore, a small shift (at least $50 \mathrm{MHz}$ ), between the resonance horizontal and vertical frequencies, is introduced in the frequency band under investigation ( 6.5 to $10.5 \mathrm{GHz}$ ).

(3) The optimization process for the remaining geometric parameters is simplified by setting all resonators to have the same slot width, $W$, with equal slot spacings. That is, there would be only two parameters to optimize with respect to slot spacings, $\Delta^{\mathrm{V}}$ and $\Delta^{\mathrm{H}}$. The different codes can be generated based on the all-one structure presented in Figure 1(a) by simply changing the lengths of two adjacent resonators. In fact, increasing the length of the shorter resonator or decreasing the length of the longer one, by an amount equal to the slot spacing, will remove the 


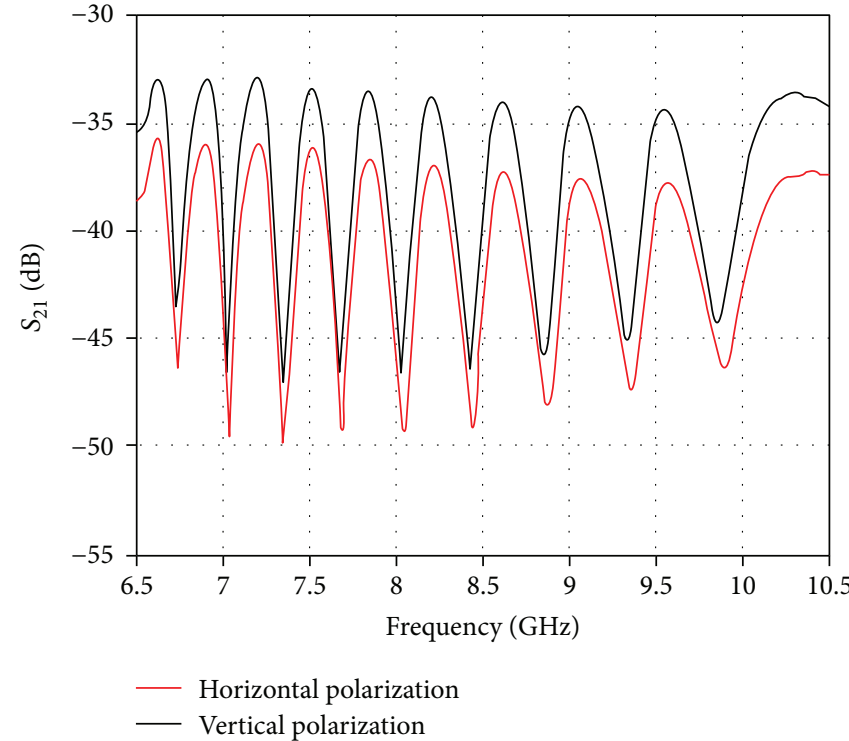

(a)

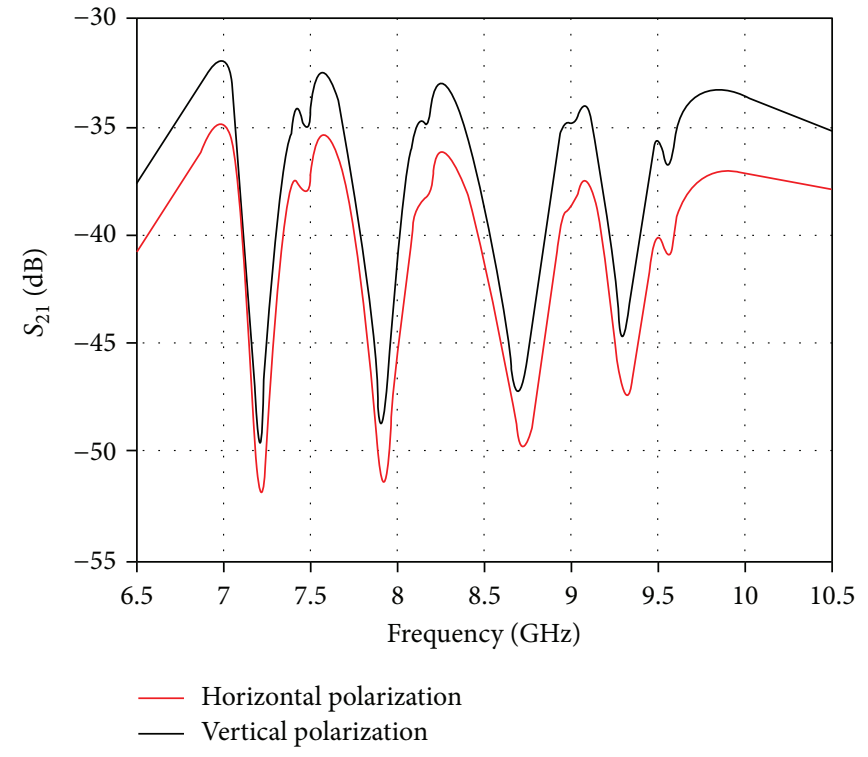

(b)

FIgURE 2: Tag-simulated response under horizontal and vertical excitations for (a) code 111111111 and (b) code 010101010.

TABle 1: Comparison of different chipless RFID tags (bistatic measurements).

\begin{tabular}{|c|c|c|c|c|c|}
\hline Resonators' type & $\begin{array}{c}\text { Frequency range } \\
(\mathrm{GHz})\end{array}$ & $\begin{array}{l}\text { Coding spatial capacity } \\
\left(\mathrm{bits} / \mathrm{cm}^{2}\right)\end{array}$ & $\begin{array}{l}\text { Coding spectral capacity } \\
\text { (bits/GHz) }\end{array}$ & $\begin{array}{l}\text { Coding density } D \\
\left(\text { bits } / \mathrm{GHz}^{*} \mathrm{~cm}^{2} \text { ) }\right.\end{array}$ & $\begin{array}{l}\text { Read range } \\
(\mathrm{cm})\end{array}$ \\
\hline C-folded dipoles [21] & $2-5$ & 1.25 & 5 & 0.416 & 80 \\
\hline SAW [22] & 2.44 & 1 & NA & NA & NA \\
\hline Delay line [23] & $3.1-10.6$ & 0.17 & 1.06 & 0.023 & 80 \\
\hline Slot [24] & $3.1-10.6$ & 4.17 & 3.2 & 0.556 & 60 \\
\hline C-shaped [25] & $2-4$ & 1.14 & 10 & 0.57 & 50 \\
\hline Stub-loaded [26] & $2.5-5.5$ & 1 & 2.66 & 0.33 & 50 \\
\hline Stepped impedance [27] & $3.1-10.6$ & 7.9 & 10.53 & 1.053 & 50 \\
\hline Slot-loaded [28] & $7-12$ & 1.47 & 3.2 & 0.29 & 5 \\
\hline Split ring resonators [29] & $2-3$ & 2.37 & 16 & 2.37 & NA \\
\hline U-shaped strip [30] & $2-4$ & 1.09 & 10 & 0.545 & 30 \\
\hline U-shaped strip [31] & $2-4$ & 0.769 & 10 & 0.385 & 25 \\
\hline Double inverted U [32] & $4-14$ & 7.73 & 2.99 & 1.546 & NA \\
\hline DP U shape [33] & $7-12$ & 16.6 & 3.2 & 3.32 & 20 \\
\hline $\begin{array}{l}\text { Nested concentric square } \\
\text { loops [34] }\end{array}$ & $8-14$ & 19 & 3 & 3.16 & 20 \\
\hline This work & $6.5-10.5$ & 18.8 & 4.5 & 4.7 & 80 \\
\hline
\end{tabular}

resonance frequency in the spectral response of the backscattered chipless RFID tag. The corresponding bit of the code will then change from 1 to 0 or vice versa. For instance, Figure 1(b) shows the tag structure for the code 010101010, in both horizontal and vertical polarizations.

\section{Simulation Results}

In Figure 2(a), we present the variation of the simulated backscattered response versus frequency from 6.5 to $10.5 \mathrm{GHz}$, under vertical (red) and horizontal (black) polarization excitations, for the nine-bit all-one tag. Ideally, for high density and capacity, the signatures under different polarizations should have the minimum possible frequency shift between corresponding nulls. Reducing the frequency shift allows the maximization of number of bits over a predefined spectrum.

By virtue of Figure 2(a), the simulated null depths are varying from $9 \mathrm{~dB}$ to $14.5 \mathrm{~dB}$. Moreover, the null separation between different bits varies from $0.288 \mathrm{GHz}$ up to $0.603 \mathrm{GHz}$. This shift increases as bit significance increases. We notice that the null bandwidth increases with the increase in frequency. Furthermore, the null depth varies and reaches $14.15 \mathrm{~dB}$ for the third null. The variations in 


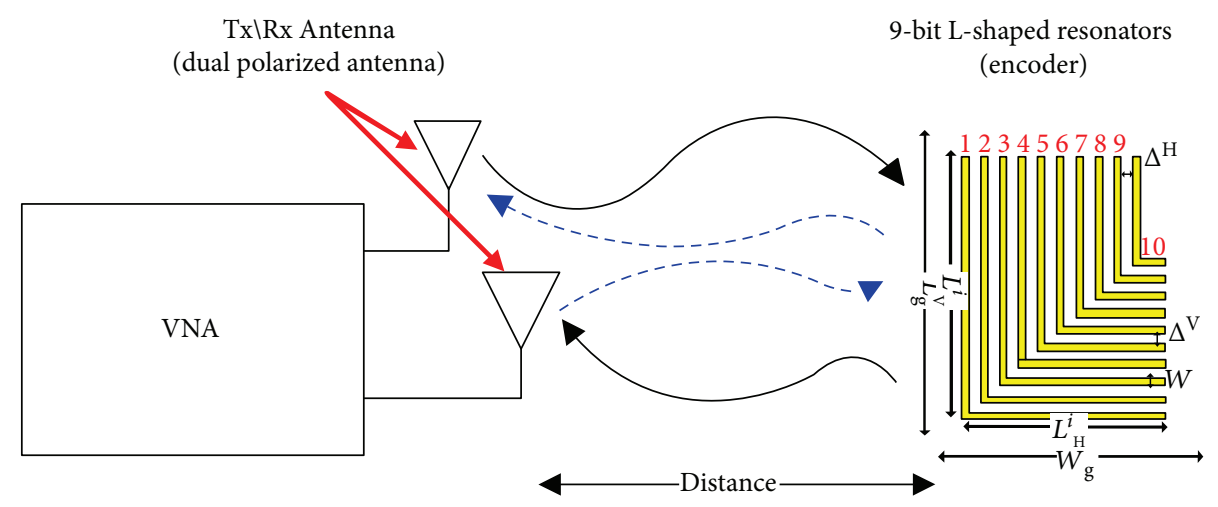

(a)

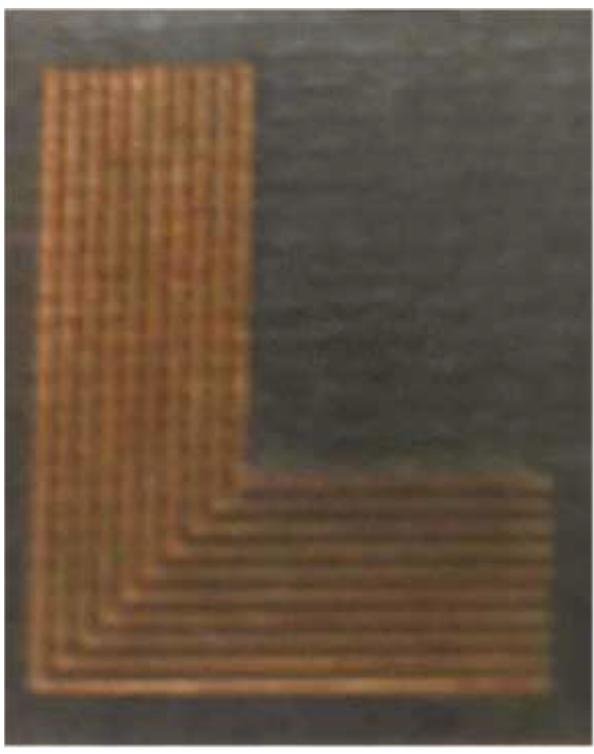

(b)

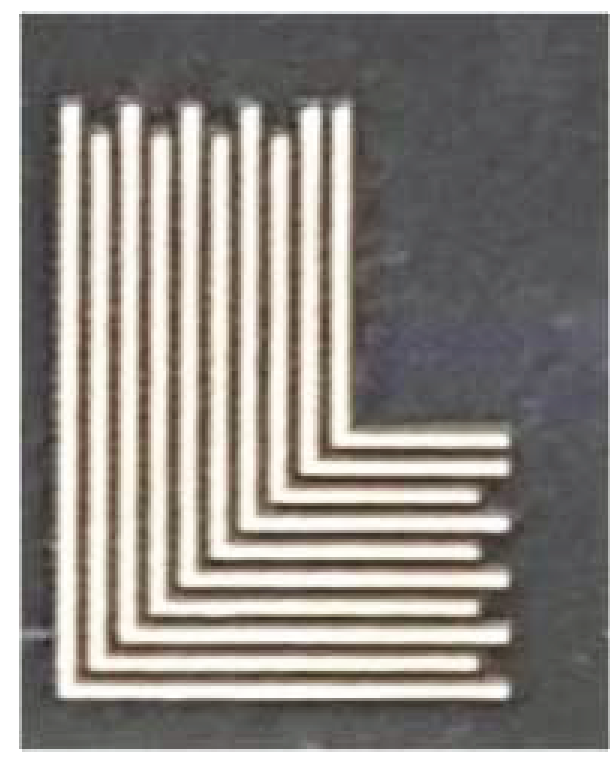

(c)

FIGURE 3: (a) Experimental setup of reader and backscattering tag, (b) fabricated chipless tag ID 111111111, and (c) fabricated chipless tag ID 010101010.

the null depth, resonant frequency, and frequency spacing are due to the periodicity of the FSS structure, which are controlled by the vertical and horizontal spacings, $\Delta^{\mathrm{V}}$ and $\Delta^{\mathrm{H}}$, respectively. Figure 2(b), on the other hand, shows the spectral responses/signatures designed for the 010101010 code. This tag has 10 resonators, and their respective responses present nulls generated by increasing or decreasing the slot horizontal length by $\Delta^{\mathrm{H}}$ and the vertical length by $\Delta^{\mathrm{V}}$ so as to eliminate or create a resonance at a lower or a higher frequency.

For comparison purposes, Table 1 reports the spectral and spatial capacities, the coding density, and read and frequency ranges of the proposed tag in contrast to other tags available in literature also designed for bistatic systems [21-34]. It is clear from the table that our proposed design outperforms the previously reported results, in terms of space and frequency band efficiencies.

\section{Experimental Results}

The proposed designs for different codes have been fabricated and measured using two linearly polarized UWB horn antennas operating from 2 to $18 \mathrm{GHz}$ and having gain values from $10 \mathrm{dBi}$ to $12 \mathrm{dBi}$. The two antennas are spaced by a $15 \mathrm{~cm}$ distance to ensure a perfect isolation. These UWB antennas are employed to examine the tag with horizontal and vertical polarizations. The schematic diagram for the experimental setup is given in Figure 3.

The measurements have been conducted in a nonanechoic environment, where the tag was placed at a $25 \mathrm{~cm}$ distance from the antennas. Figure 4(a) shows the measured responses of the two fabricated codes of the L-shaped chipless RFID tag under vertical and horizontal polarization excitations. The resonator's notch depth variation is observed from $6.2 \mathrm{~dB}$ to $12.8 \mathrm{~dB}$ and from $7.5 \mathrm{~dB}$ to $15.1 \mathrm{~dB}$ for horizontal and vertical polarizations, respectively.

The null bandwidth is smaller than $320 \mathrm{MHz}$ for all bits. Referring to Figures 2 and 4, a quite reasonable agreement is observed between the measured and simulated results except for a small shift in frequency, which may be due to fabrication tolerance.

Figure 5 presents the measurements of the backscattered signature of code 010101010 as a function of the 


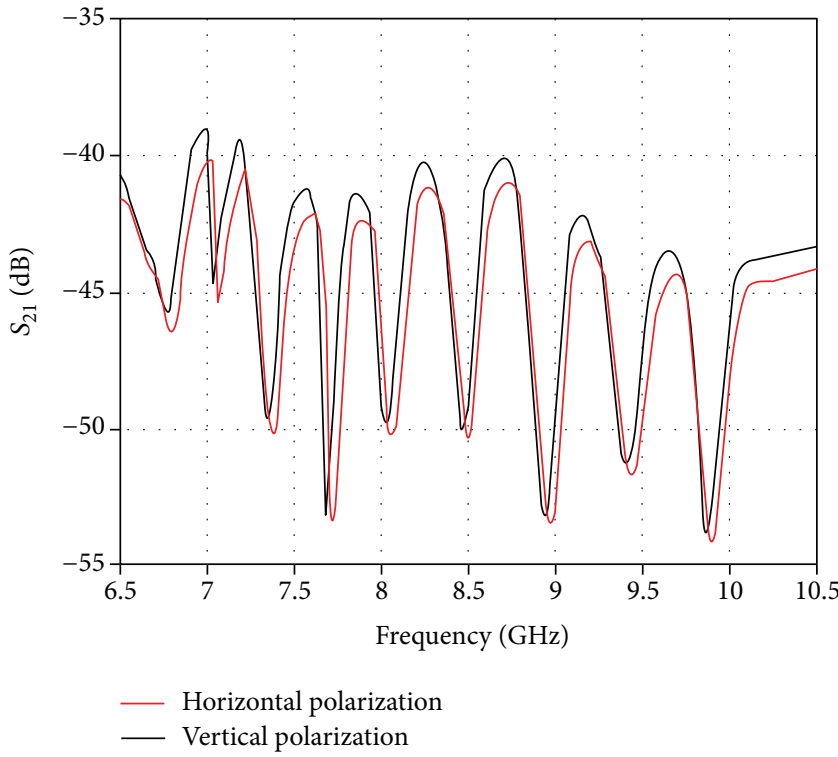

(a)

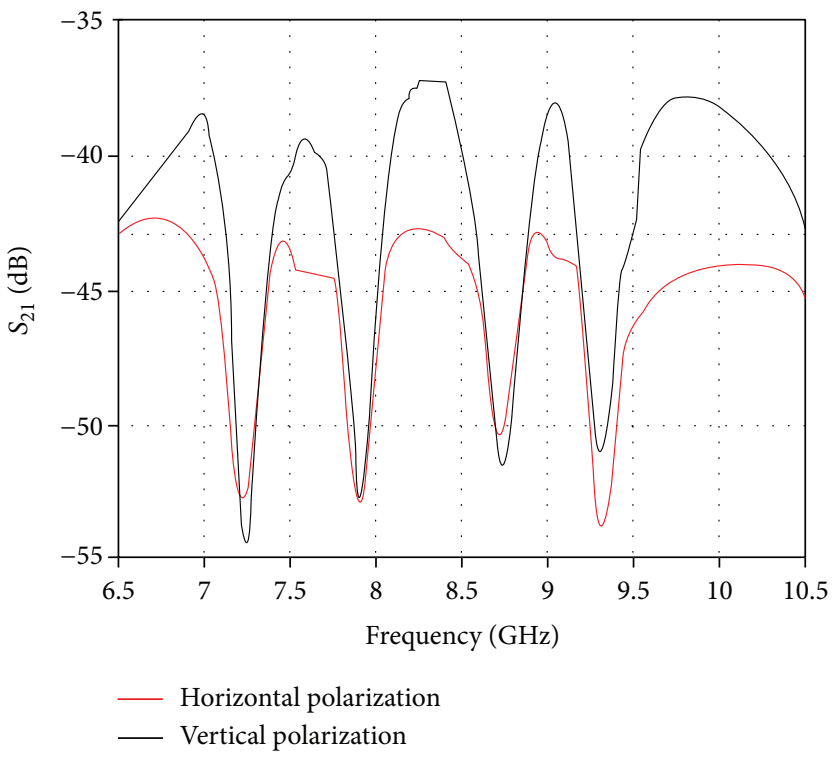

(b)

Figure 4: Tag-measured response under horizontal and vertical excitation for (a) code 111111111 and (b) code 010101010.

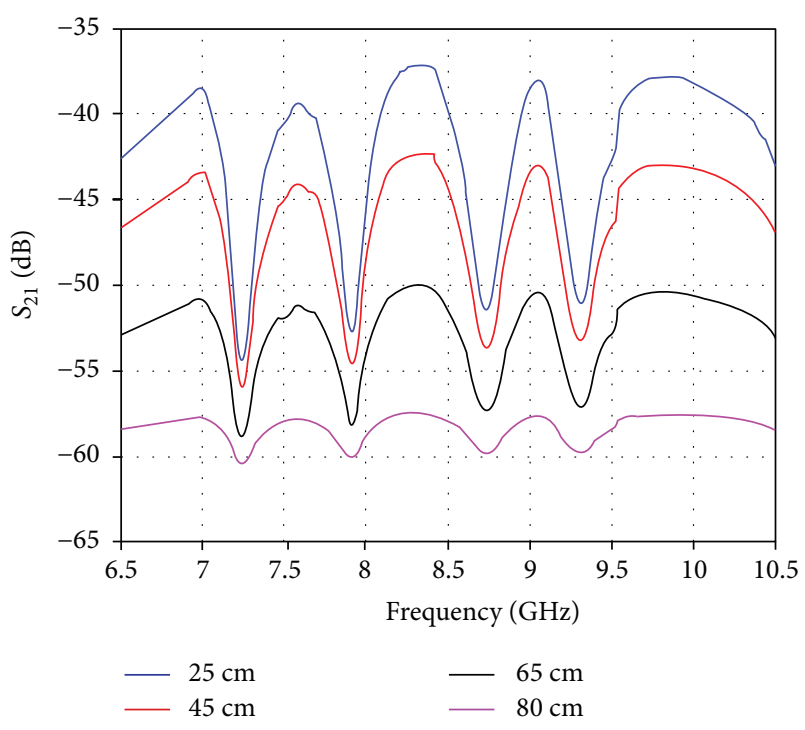

Figure 5: Measured frequency response versus distance for code 010101010 under horizontal polarization.

tag-reader distance. A read-range starting from $25 \mathrm{~cm}$ up to $80 \mathrm{~cm}$ is achieved. Therefore, the maximum read-range is $80 \mathrm{~cm}$ for this experimental setup, after which the signal deteriorates.

\section{Code Identification}

The proposed backscattering codes have well-defined signatures in the frequency domain. Therefore, we consider the frequency domain for their identification.
Because the spectral nulls pertaining to the horizontal and vertical polarizations of the same code are separated by small frequency shifts $(10-90 \mathrm{MHz})$, it is important that the frequency response of a code is to be preprocessed such as the differences between the responses of horizontal and vertical polarizations become more pronounced. Figure 6 shows the proposed identification system. The frequency response of the backscattered signal is first computed and then passed through a first-order differencing filter whose transfer function is defined in (3) as follows:

$$
H(z)=1-z^{-1}
$$

This filter is followed by a polarity correlator, the output of which is normalized to obtain the cross-correlation coefficient whose values lie between +1 and -1 . Note that smaller values of the cross-correlation coefficient (close to zero) mean corresponding signals are more distinguishable from each other. Let $c_{i}(n)$ and $c_{j}(n)$ denote sequences representing the responses pertaining to the $i$ th and $j$ th codes. The crosscorrelation coefficient, $\gamma_{i j}$, between these two responses (sequences) is defined in (4) as follows [36].

$$
\gamma_{i j}=\frac{\sum_{n} c_{i}(n) c_{j}(n)}{\sum_{n} c_{i}^{2}(n) \times \sum_{n} c_{j}^{2}(n)} .
$$

Figures 7 and 8 show output of the horizontal and vertical components of code 111111111 and 010101010, computed after the filter and at the output of the polarity correlator. The output of the filter clearly shows the shifts between the horizontal and vertical components, but may have spikes introduced due to the differencing operation, which is equivalent to the differentiation operation applied to continuous types of signals. These spikes, which appear at nulls' locations, may bias the computation of the correlation coefficient 


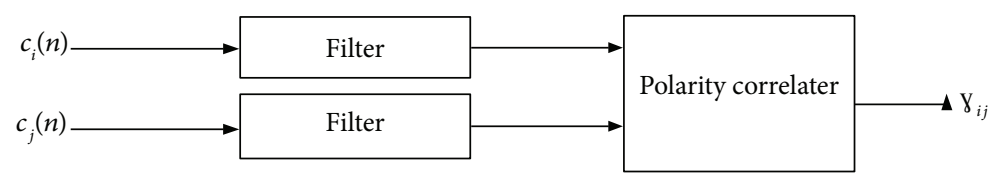

Figure 6: The proposed identification system.

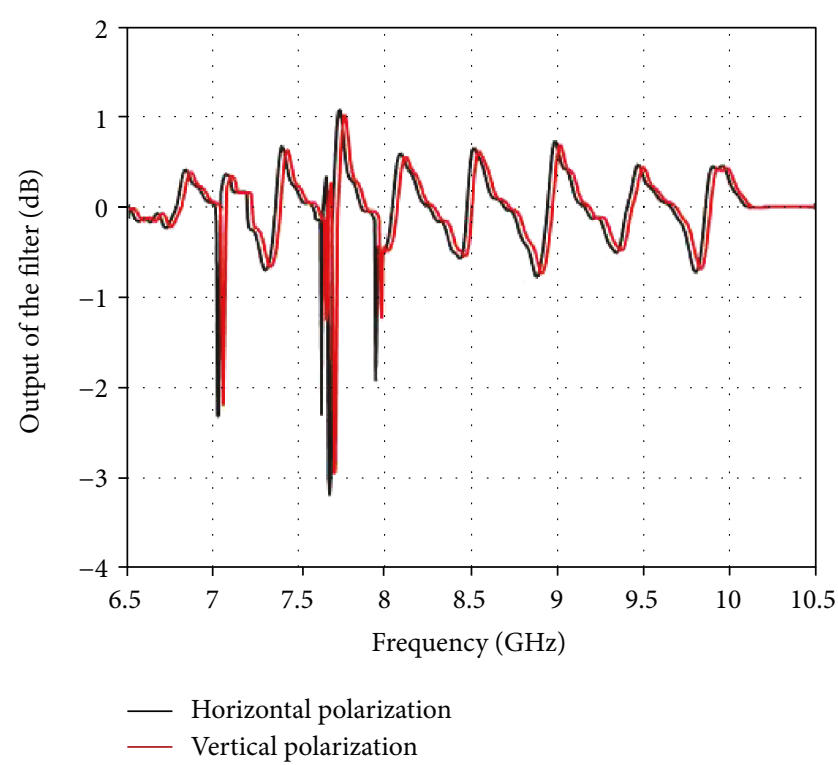

(a)

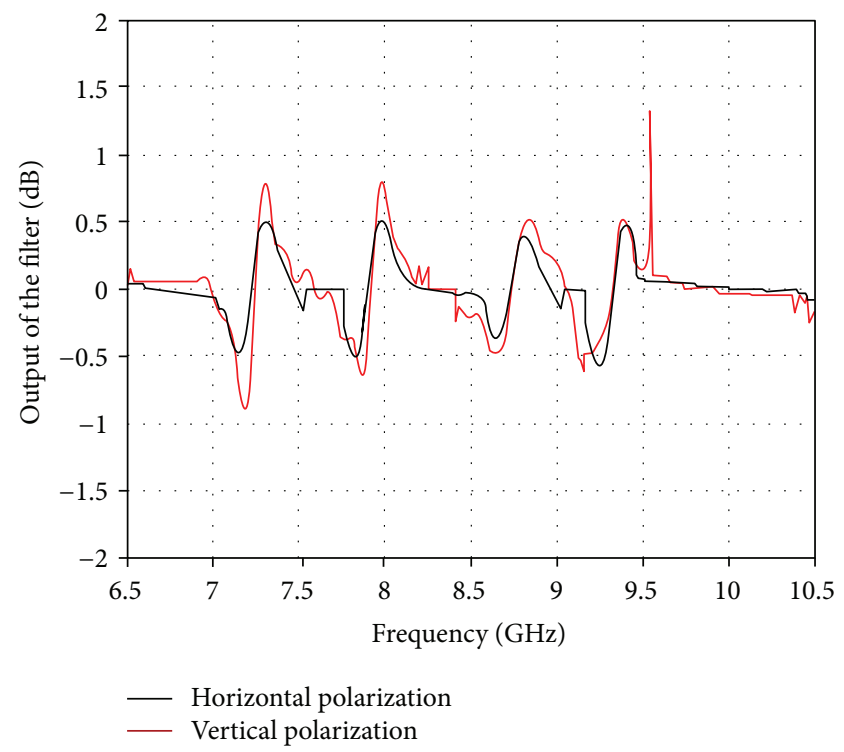

(b)

FIgURE 7: The filter output for the horizontal and vertical components of (a) code 111111111 and (b) code 010101010.

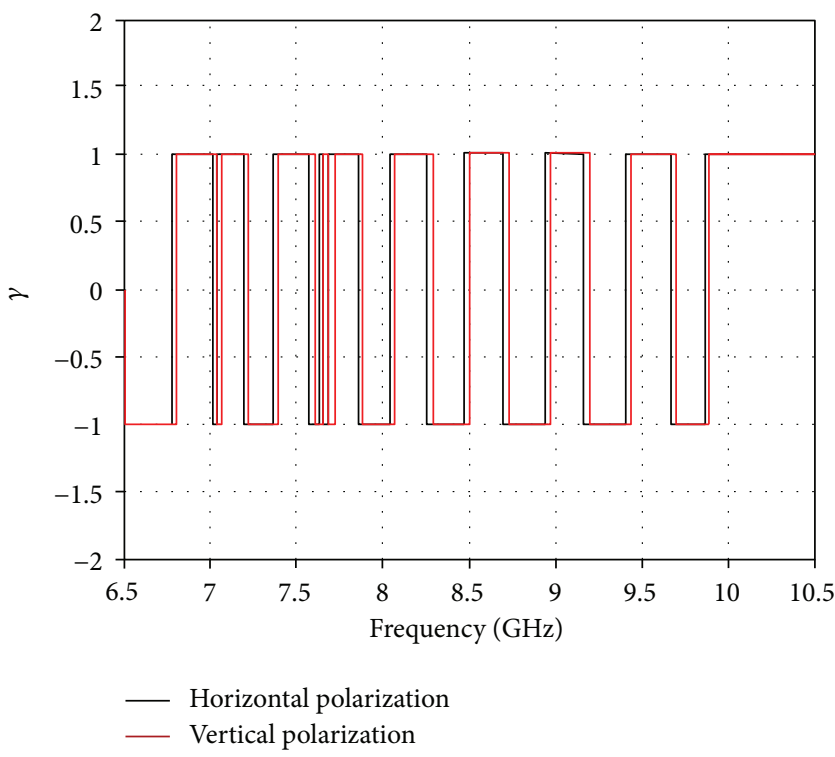

(a)

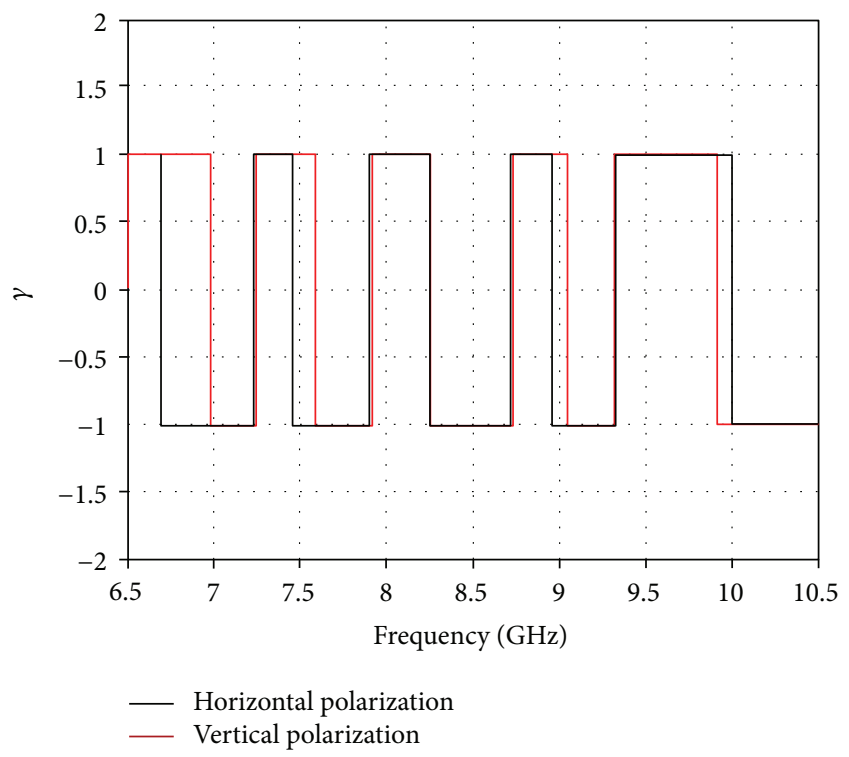

(b)

FIGURE 8: The output of the polarity correlator for the horizontal and vertical components of codes 111111111 and 010101010 , respectively. 
TABLE 2: The correlation coefficient between the horizontal and vertical components of codes 11111111 and 010101010 after polarity correlator.

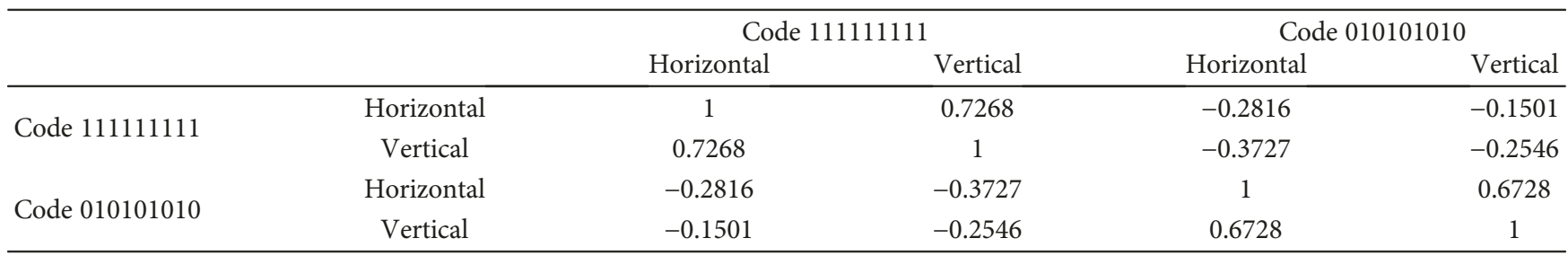

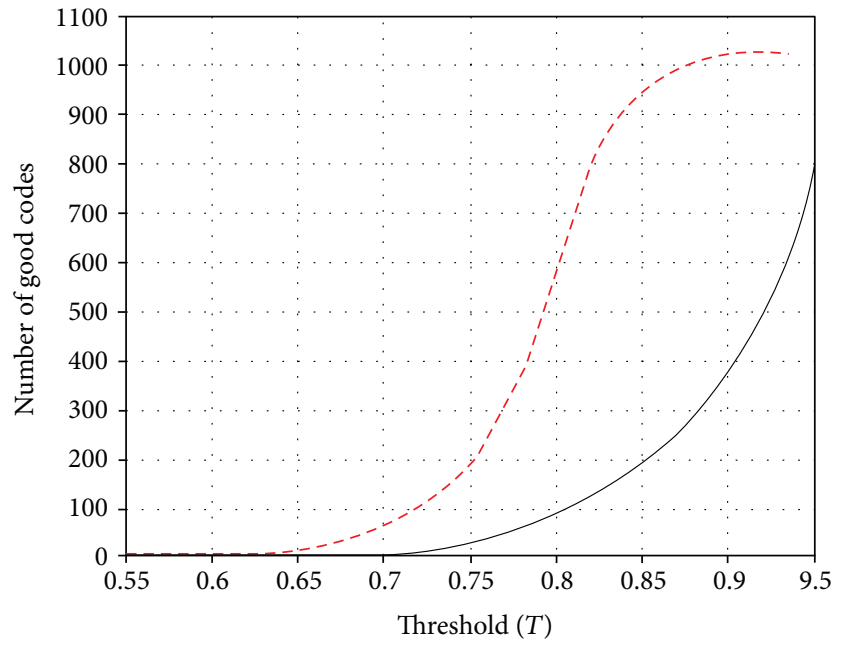

- Output of the filter

- - - Output of the polarity corelator

FIGURE 9: The binary codes whose correlation coefficient is less than the threshold $T$ for the output of the filter and the polarity correlator.

because of their relatively large values. Therefore, dealing with the polarity of the filter's output removes this pitfall. Further, the resulting binary $( \pm 1)$ sequence still preserves distinguishing features, which can be exploited for identification.

This observation is confirmed by the results of Table 2, which shows the correlation coefficients pertaining to the horizontal and vertical components of the two codes 111111111 and 010101010. The computations are applied to sequences extracted from codes' responses measured at $25 \mathrm{~cm}$. By virtue of Table 2, it is obvious that the similarity between different codes is insignificant.

However, the correlation coefficient between the horizontal and vertical components of the same code may go as high as 0.7268 . For further analysis, the frequency responses of all 9 bits have been generated by simulation. The number of resulting codes is 1024 (512 codes have horizontal polarization, and another 512 codes have vertical polarization).

Figure 9 shows the number of codes whose correlation coefficient is less than $T$, where $T$ has values between 0.55 and 1. The figure shows the results of two cases: the first is when the correlation coefficient is computed right after the filter, while the second is for the output of the polarity correlator. It is obvious that the polarity correlator is more efficient. Note that among the 1024 codes, there are 175, 984, and 1021 binary codes having correlation coefficients less than or equal to $0.75,0.85$, and 0.90 , respectively. In other words, it can be stated that the responses of binary codes can produce distinguishable sequences. Based on the application at hand, it would be possible to generate a reasonable number of binary codes from a 9-bit resonator. The binary codes can be selected so as to maintain the similarity between any two codes below a predefined threshold. In any event, a code is detected by determining the maximum correlation coefficient of this unknown code with all reference codes.

\section{Conclusion}

A dual polarized chipless RFID tag operating from 6.5 to $10.5 \mathrm{GHz}$ is presented. L-shaped resonators are introduced to increase the bit-encoding capacity over a unit area. The key performance parameters are optimized with several full-wave simulations to have sharp resonant notches of $14.5 \mathrm{~dB}$ with a bandwidth smaller than $320 \mathrm{MHz}$. The simulation performance shares reasonable agreement with the measured performance. The proposed L-shaped FD tag operates over a $4 \mathrm{GHz}$ bandwidth from 6.5 to $10.5 \mathrm{GHz}$. Using the proposed structure, it was possible to achieve an encoding density of $4.7 \mathrm{bits} / \mathrm{GHz} / \mathrm{cm}^{2}$ which is, to the best of our knowledge, the largest encoding density of a chipless RFID tag reported so far. The design of the proposed RFID tag can easily be modified to generate new codes, simply by varying the resonator's lengths by an amount equal to the slots' spacing. A signal processing technique based on correlation analysis has been also introduced for the detection of an unknown code. This technique adopts a polarity scheme to process frequency domain responses, which will not only enable higher number of distinguishable codes but also provide a fast method for inexpensive computation.

\section{Data Availability}

All the data supporting the results were shown in the paper and can be applicable from the corresponding author.

\section{Conflicts of Interest}

The authors declare no conflict of interest. 


\section{Acknowledgments}

The authors extend their appreciation to the Deanship of Scientific Research at King Saud University for funding this work through research group No. RG-1438-092.

\section{References}

[1] E. Perret, Radio Frequency Identification and Sensors From RFID to Chipless RFID, John Wiley and Sons, Hoboken, NJ, USA, 2014.

[2] S. Preradovic and N. C. Karmakar, "Chipless RFID: bar code of the future," IEEE Microwave Magazine, vol. 11, no. 7, pp. 87-97, 2010.

[3] M. Popperl, A. Parr, C. Mandel, R. Jakoby, and M. Vossiek, "Potential and practical limits of time-domain reflectometry chipless RFID," IEEE Transactions on Microwave Theory and Techniques, vol. 64, no. 9, pp. 2968-2976, 2016.

[4] M. M. Khan, F. A. Tahir, M. F. Farooqui, A. Shamim, and H. M. Cheema, "3.56-bits $/ \mathrm{cm}^{2}$ compact inkjet printed and application specific chipless RFID tag," IEEE Antennas and Wireless Propagation Letters, vol. 15, pp. 1109-1112, 2016.

[5] A. Habib, Y. Amin, M. A. Azam, J. Loo, and H. Tenhunen, "Frequency signatured directly printable humidity sensing tag using organic electronics," IEICE Electronics Express, vol. 14, no. 3, article 20161081, 2017.

[6] S. Preradovic, I. Balbin, N. C. Karmakar, and G. F. Swiegers, "Multiresonator-based chipless RFID system for low-cost item tracking," IEEE Transactions on Microwave Theory and Techniques, vol. 57, no. 5, pp. 1411-1419, 2009.

[7] A. Vena, E. Perret, and S. Tedjini, "Chipless RFID tag using hybrid coding technique," IEEE Transactions on Microwave Theory and Techniques, vol. 59, no. 12, pp. 3356-3364, 2011.

[8] A. Lazaro, A. Ramos, D. Girbau, and R. Villarino, "Signal processing techniques for chipless UWB RFID thermal threshold detector detection," IEEE Antennas and Wireless Propagation Letters, vol. 15, pp. 618-621, 2016.

[9] S. Shrestha, M. Balachandran, M. Agarwal, V. V. Phoha, and K. Varahramyan, "A chipless RFID sensor system for cyber centric monitoring applications," IEEE Transactions on Microwave Theory and Techniques, vol. 57, no. 5, pp. 1303-1309, 2009.

[10] A. Lazaro, A. Ramos, D. Girbau, and R. Villarino, "Chipless UWB RFID tag detection using continuous wavelet transform," IEEE Antennas and Wireless Propagation Letters, vol. 10, pp. 520-523, 2011.

[11] S. Dey, J. K. Saha, and N. C. Karmakar, "Smart sensing: chipless RFID solutions for the internet of everything," IEEE Microwave Magazine, vol. 16, no. 10, pp. 26-39, 2015.

[12] K. V. S. Rao, P. V. Nikitin, and S. F. Lam, “Antenna design for UHF RFID tags: a review and a practical application," IEEE Transactions on Antennas and Propagation, vol. 53, no. 12, pp. 3870-3876, 2005.

[13] T. Dissanayake and K. P. Esselle, "Prediction of the notch frequency of slot loaded printed UWB antennas," IEEE Transactions on Antennas and Propagation, vol. 55, no. 11, pp. 3320-3325, 2007.

[14] S. H. Zainud-Deen et al., "Dual-polarized chipless RFID tag with temperature sensing capability," in 2014 31st National Radio Science Conference (NRSC), Cairo, Egypt, April 2014.
[15] F. Costa, A. Gentile, S. Genovesi et al., "A depolarizing chipless RF label for dielectric permittivity sensing," IEEE Microwave and Wireless Components Letters, vol. 28, no. 5, pp. 371-373, 2018.

[16] M. P. Jayakrishnan, A. Vena, B. Sorli, and E. Perret, "Solidstate conductive-bridging reconfigurable RF-encoding particle for chipless RFID applications," IEEE Microwave and Wireless Components Letters, vol. 28, no. 6, pp. 506-508, 2018.

[17] D. Laila, R. Thomas, C. M. Nijas, and P. Mohanan, “A novel polarization independent chipless RFID tag using multiple resonators," Progress In Electromagnetics Research Letters, vol. 55, pp. 61-66, 2015.

[18] V. R. Sajitha, C. M. Nijas, T. K. Roshna, K. Vasudevan, and P. Mohanan, "Compact cross loop resonator based chipless RFID tag with polarization insensitivity," Microwave and Optical Technology Letters, vol. 58, no. 4, pp. 944-947, 2016.

[19] F. Costa, S. Genovesi, and A. Monorchio, "Normalization-free chipless RFIDs by using dual-polarized interrogation," IEEE Transactions on Microwave Theory and Techniques, vol. 64, no. 1, pp. 310-318, 2016.

[20] A. Vena, E. Perret, and S. Tedjini, "High-capacity chipless RFID tag insensitive to the polarization," IEEE Transactions on Antennas and Propagation, vol. 60, no. 10, pp. 4509-4515, 2012.

[21] O. Rance, R. Siragusa, P. Lemaitre-Auger, and E. Perret, “Toward RCS magnitude level coding for chipless RFID," IEEE Transactions on Microwave Theory and Techniques, vol. 64, no. 7, pp. 2315-2325, 2016.

[22] C. S. Hartmann, "A global SAW ID tag with large data capacity," in 2002 IEEE Ultrasonics Symposium, 2002. Proceedings, pp. 65-699, Munich, Germany, October 2002.

[23] B. Shao, Q. Chen, Y. Amin, S. M. David, R. Liu, and L.-R. Zheng, "An ultra-low-cost RFID tag with 1.67 gbps data rate by ink-jet printing on paper substrate," in 2010 IEEE Asian Solid-State Circuits Conference, pp. 1-4, Beijing, China, November 2010.

[24] R. Rezaiesarlak and M. Manteghi, "Complex-naturalresonance-based design of chipless RFID tag for high-density data," IEEE Transactions on Antennas and Propagation, vol. 62, no. 2, pp. 898-904, 2014.

[25] A. Vena, E. Perret, and S. Tedjini, "A fully printable chipless RFID tag with detuning correction technique," IEEE Microwave and Wireless Components Letters, vol. 22, no. 4, pp. 209-211, 2012.

[26] D. Girbau, J. Lorenzo, A. Lazaro, C. Ferrater, and R. Villarino, "Frequency-coded chipless RFID tag based on dual-band resonators," IEEE Antennas and Wireless Propagation Letters, vol. 11, pp. 126-128, 2012.

[27] C. M. Nijas, U. Deepak, P. V. Vinesh et al., "Low-cost multiplebit encoded chipless RFID tag using stepped impedance resonator," IEEE Transactions on Antennas and Propagation, vol. 62, no. 9, pp. 4762-4770, 2014.

[28] M. A. Islam and N. C. Karmakar, "A novel compact printable dual-polarized chipless rfid system," IEEE Transactions on Microwave Theory and Techniques, vol. 60, no. 7, pp. 21422151, 2012.

[29] C. Herrojo, F. Paredes, J. Mata-Contreras, S. Zuffanelli, and F. Martin, "Multistate multiresonator spectral signature barcodes implemented by means of S-shaped split ring resonators (S-SRRS)," IEEE Transactions on Microwave Theory and Techniques, vol. 65, no. 7, pp. 2341-2352, 2017. 
[30] M. Polivka, J. Havlicek, M. Svanda, and J. Machac, "Improvement in robustness and recognizability of RCS response of U-shaped strip-based chipless RFID tags," IEEE Antennas and Wireless Propagation Letters, vol. 15, pp. 2000-2003, 2016.

[31] M. Svanda, M. Polivka, J. Havlicek, and J. Machac, "Chipless RFID tag with an improved magnitude and robustness of RCS response," Microwave and Optical Technology Letters, vol. 59, no. 2, pp. 488-492, 2017.

[32] N. Javed, A. Habib, A. Akram, Y. Amin, and H. Tenhunen, "16-bit frequency signatured directly printable tag for organic electronics," IEICE Electronics Express, vol. 13, article 20160406, 2016

[33] M. A. Islam and N. C. Karmakar, "Real-world implementation challenges of a novel dual-polarized compact printable chipless RFID tag," IEEE Transactions on Microwave Theory and Techniques, vol. 63, no. 12, pp. 4581-4591, 2015.

[34] H.-f. Huang and L. Su, "A compact dual-polarized chipless RFID tag by using nested concentric square loops," IEEE Antennas and Wireless Propagation Letters, vol. 16, pp. 10361039, 2017.

[35] K. Issa, M. A. Ashraf, M. R. AlShareef, H. Behairy, S. Alshebeili, and H. Fathallah, "A novel $L$-shape ultra wideband chipless radio-frequency identification tag," International Journal of Antennas and Propagation, vol. 2017, Article ID 2823565, 7 pages, 2017.

[36] M. H. Hayes, Statistical Digital Signal Processing and Modeling, John Wiley \& Sons, 2009. 


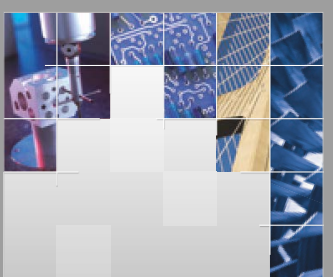

\section{Enfincering}
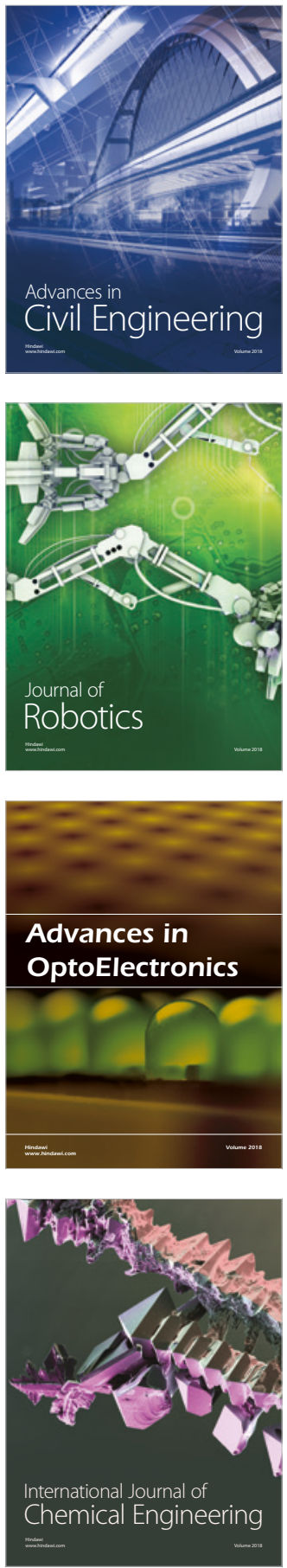

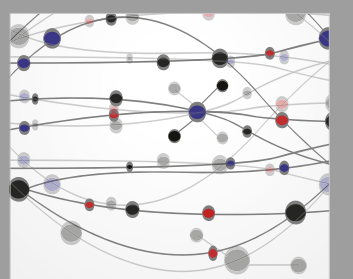

\section{Rotating \\ Machinery}

The Scientific World Journal

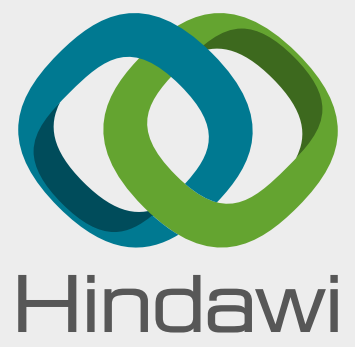

Submit your manuscripts at

www.hindawi.com
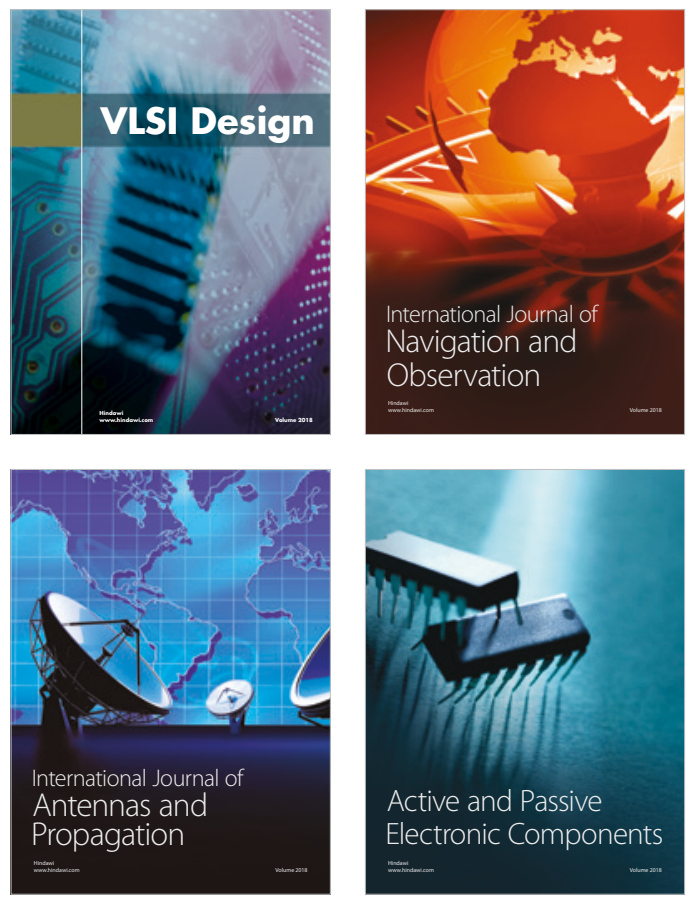
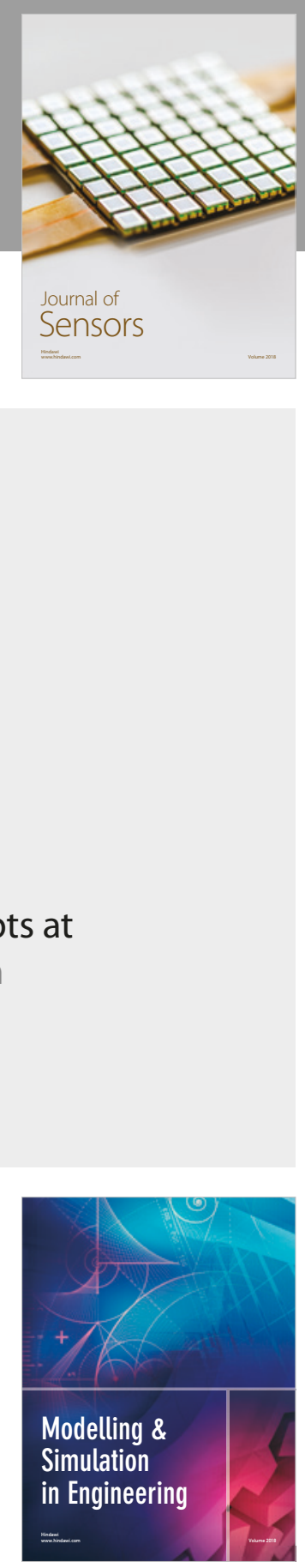

\section{Advances \\ Multimedia}
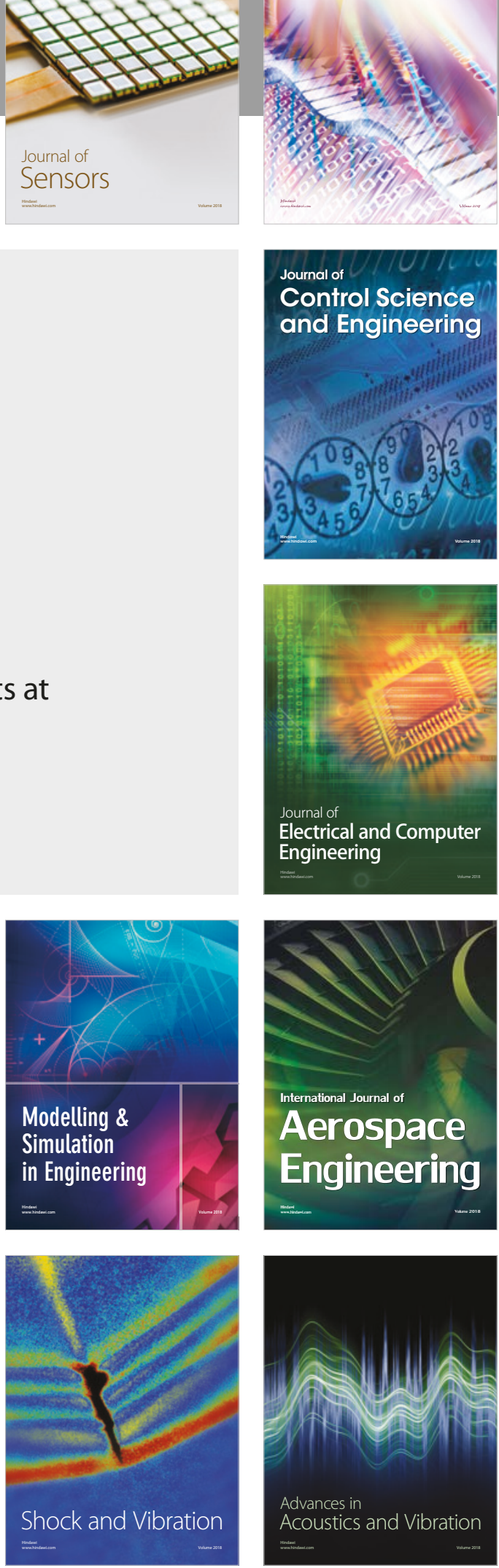